\title{
iccafd
}

Revista Iberoamericana de Ciencias de la Actividad Física y el Deporte

\section{EFECTOS DE LA MICROGRAVEDAD SOBRE LA CONDICIÓN FÍSICA EN ASTRONAUTAS. ¿EXISTE INTERVENCIÓN FISIOTERAPÉUTICA?}

\section{EFFECTS OF MICROGRAVITY ON PHYSICAL CONDITION IN ASTRONAUTS. DOES PHYSIOTHERAPEUTIC INTERVENTION EXIST?}

Alfonso Mantilla, José Iván ${ }^{1}$ y Martínez Santa, Jaime ${ }^{2}$

1 Alfonso Mantilla, José Iván. Fisioterapeuta. Universidad del Rosario. Colombia. josealfonso25@hotmail.com

2 Martínez Santa, Jaime. Fisioterapeuta. Especialista en epidemiologia y estadística. Universidad Nacional. Colombia. jmartinezsanta@yahoo.com

Código UNESCO: 321311 . Fisioterapia.

Clasificación Consejo de Europa: 14. Fisioterapia y rehabilitación.

Recibido el 17 de abril de 2018

Aceptado el 19 de junio de 2018

Correspondencia:

Alfonso Mantilla, José Iván

josealfonso25@hotmail.com

DOI: http://dx.doi.org/10.24310/riccafd.2018.v7i2.5089

\section{RESUMEN}

La fisioterapia como el área del estudio del movimiento corporal humano ha abierto caminos en investigación en distintos ámbitos. Por ejemplo, en medicina aeroespacial se ha reportado que la exposición en el espacio durante una misión supone cambios fisiológicos a nivel cardiovascular, osteomuscular y neuromuscular en los astronautas debido a la exposición a microgravedad, se ha reportado que protocolos de actividad física y ejercicio pueden ser utilizados para la prevención y entrenamiento de astronautas mostrando efectos significativos en la condición física.

PALABRAS CLAVE: astronautas, vuelos espaciales, medicina aeroespacial, aviación. 


\begin{abstract}
Physiotherapy as the area of study of human body movement has opened up avenues of research in different fields. For example, in aero-spatial medicine it has been reported that space exposure during a mission involves physiological changes in cardiovascular, musculoskeletal and neuromuscular levels in astronauts due to exposure to microgravity, it has been reported that physical activity and exercise protocols can be used for the prevention and training of astronauts showing significant effects on physical condition.
\end{abstract}

KEY WORDS: astronauts, space flight, aero-spatial medicine, aviation

\title{
INTRODUCCIÓN
}

La medicina aeroespacial tiene sus inicios en el año 1917 en la base de operaciones militares de San Antonio en los Estados Unidos de América, en el año 1926 esta se convierte en la escuela de medicina de aviación donde se comienza a vislumbrar la importancia de la investigación en medicina aeroespacial. En el año 1969 se realiza una cooperación con la NASA enfocando los estudios en investigaciones en misiones aeronáuticas y militares ${ }^{(1)}$. Se ha reportado que la exposición en el espacio durante una misión supone cambios fisiológicos en los astronautas debido a la exposición a la microgravedad. Adicionalmente, después del regreso a la tierra los astronautas presentan desacondicionamiento muscular, intolerancia ortostática, desmineralización ósea, atrofia muscular y alteraciones neurovestibulares ${ }^{(2)}$.

Actualmente en los Estados Unidos de América se están diseñando protocolos para aumentar el desempeño muscular, capacidad aeróbica y resistencia con el fin de preparar a los astronautas para misiones aeroespaciales, estos se basan en ejercicios en banda sin fin, cicloergómetro y ejercicios concéntricos enfocados en musculatura de tren inferior y tronco ${ }^{(2)}$. Se ha reportado que una duración en el espacio mayor a seis meses produce cambios sustanciales a nivel muscular, esquelético y cardiopulmonar ${ }^{(3,4)}$. El objetivo de este artículo es realizar una revisión sistemática de la literatura acerca de los efectos fisiológicos causados por la exposición a la microgravedad y si existe una intervención fisioterapéutica en la rama de la medicina aeroespacial basada en ejercicio físico.

\section{MATERIAL Y METODOS}

Se realizó un estudio de revisión de la literatura en la cual se realizo una revisión de evidencia, en la cual:

Se determinaron los siguientes criterios de inclusión y exclusión. Criterios de Inclusión: Estudios contemplados del año 2000 al 2016, literatura que contemplara los siguientes términos MESH: astronauts, space flight, aerospace medicine y aviation, la literatura se aceptaría en idioma español, inglés, portugués y ruso. Se estableció como otro criterio de inclusión que la búsqueda 
de evidencia se basara en: Fuentes primarias: Estudios ECA, metanalisis, casos y controles, estudios de cohorte, revisiones sistemáticas, revisiones de literatura. Fuentes secundarias: Monografías, tesis de grado, libros. Criterios de Exclusión: estudios que su año de publicación fuera inferior al año 2000, que no contemplaran los términos MeSH establecidos, estudios que no estuvieran disponibles o completos. Se realizó una búsqueda en estas bases de datos: «Ebsco», «Pedro», «Hinari», «Elsevier», «Science Direct», «Springer», «Medline».

\section{RESULTADOS}

Dentro de la revisión de la evidencia se pudo identificar evidencia en relación a los efectos fisiológicos de la estancia prolongada en el espacio y programas de entrenamiento para astronautas. A continuación, en la figura 1 se muestra el diagrama de flujo que presenta el proceso para la selección de la literatura que se incluye en la revisión.

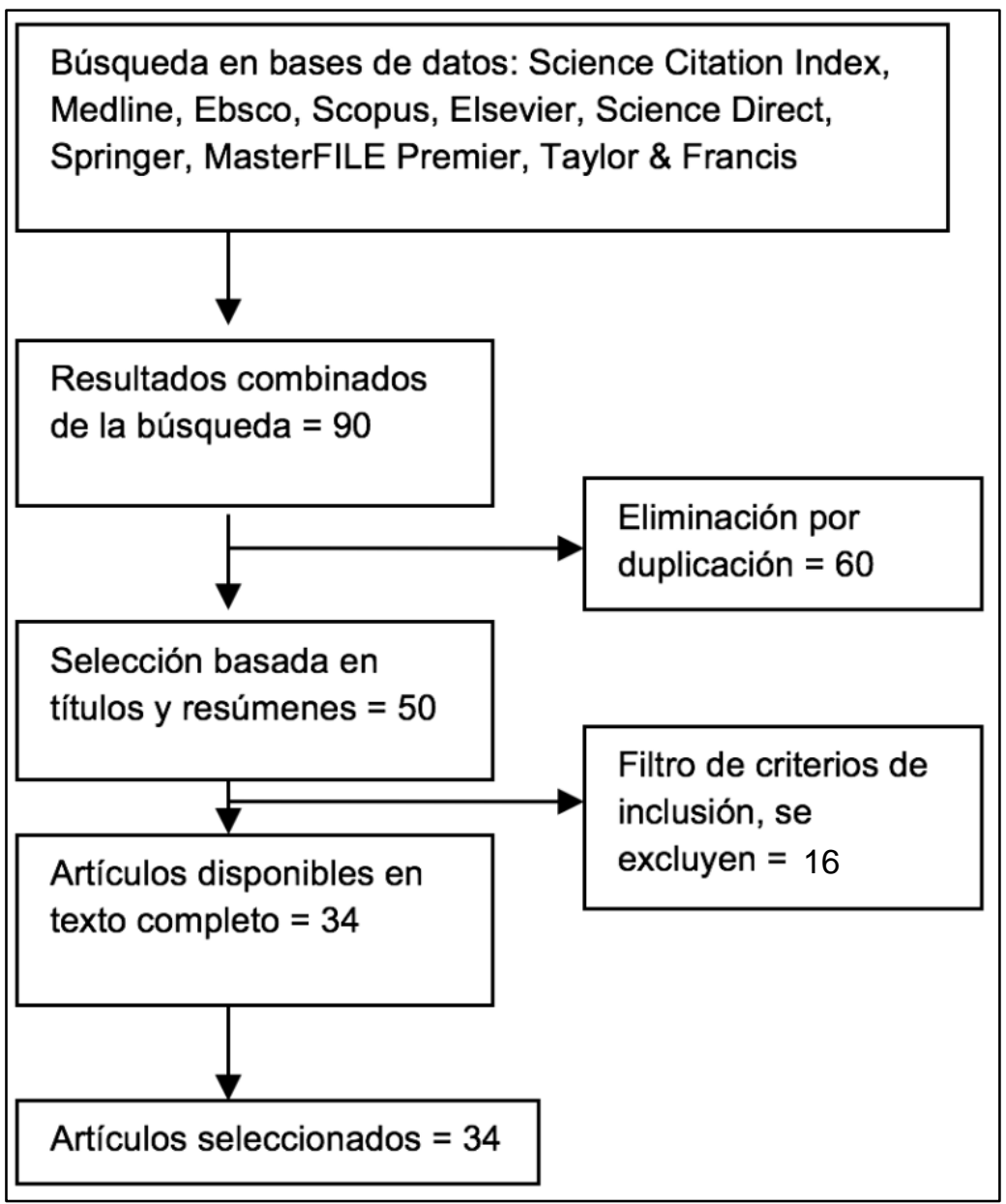

Figura 1. (Flujograma del proceso de selección de la literatura). Fuente de elaboración propia 2016.

Se pudo identificar dentro de la literatura evidencia en relación a las características de la microgravedad que causan alteraciones específicas en 
sistemas específicos del cuerpo humano y programas de entrenamiento enfocados en la prevención y entrenamiento de astronautas

\section{CARACTERÍSTICAS AEROESPACIALES}

Existen características del ambiente aeroespacial las cuales son las determinantes en los cambios fisiológicos, estas son la presurización, las fuerzas $G$ y la microgravedad. La presurización es el bombeo continuo de aire que garantiza confort y estabilidad pulmonar. Las fuerzas $G$ son la medida de aceleración y desaceleración relacionadas con la presión atmosférica; la microgravedad hace referencia a la ausencia de fuerza de gravedad ${ }^{(4)}$.

\section{ALTERACIONES FISIOLOGICAS}

\section{Ventilación, respiración e intercambio gaseoso}

Se pudo evidenciar que se produce una vasoconstricción la cual produce alteración en índices de hemoglobina, oxihemoglobina y saturación de oxígeno. Adicionalmente, la exposición a fuerzas G produce atelectasia por aceleración, neumotórax, edema pulmonar y trombosis venosa profunda ${ }^{(5-8)}$.

\section{Desempeño muscular}

Después de una larga exposición a un ambiente espacial se produce disminución de la fuerza muscular producida por atrofia con alteración del control neuromuscular y la estabilidad lumbopélvica. Adicionalmente, se produce un imbalance muscular causado por la hipertrofia de la musculatura flexora y la atrofia de los extensores lumbares lumbopélvicos ${ }^{(9-11)}$.

\section{Integridad sensorial}

Se produce una alteración entre la interpretación del cerebro de señales enviadas desde el sistema nervioso simpático. Adicionalmente, puede ocurrir una falla vestibular súbita llevando a una hipofunción de un laberinto lo cual genera el vértigo y un nistagmus vestibular. Se ha reportado una alteración conocida como la enfermedad espacial de movimiento la cual es la alteración del sistema vestibular cuando es sometido a un ambiente de ingravidez la cual produce mareo durante la producción de movimiento ${ }^{(12-16)}$.

\section{Características antropométricas}

Se produce atrofia muscular producida por la microgravedad la cual genera alteración de músculos extensores y flexores alterando las cadenas musculares anteriores y cruzadas. Consecuentemente, se produce una alteración del índice de masa corporal aumentando el peso corporal ${ }^{(17,18)}$.

\section{Ergonomía y mecánica corporal}

Los pilotos y astronautas están sometidos a dolor cervical y fatiga muscular producida por estancias prolongadas y alteraciones en el centro de 
gravedad sometido a microgravedad. Los cambios biomecánicos son dados por imbalances y bloqueos en las cadenas musculares anteriores y posteriores alterando las reacciones de soporte y la no actividad antigravitatoria $(19,20)$.

\section{Circulación}

La hipoxia no producen alteraciones graves de los niveles de lgA y niveles de cortisol, en contraste se ve una alteración de los linfocitos como una respuesta transitoria ante la regulación de las células CD69 y CD14 y una diminución de la HLA-DR ${ }^{(21) .}$

\section{PROTOCOLOS DE ENTRENAMIENTO}

Después de las misiones a nivel espacial se producen alteraciones a nivel musculoesquelético y cardiovascular. En consecuencia se han desarrollado protocolos de evaluación y entrenamiento de astronautas. Para la evaluación se tiene en cuenta entre otros; test de agilidad, sit and reach, push ups, pull ups, sliding crunches, bench press, leg press ${ }^{(22)}$. Diversas agencias internacionales han propuesto modelos de entrenamiento físico después de misiones en el espacio con el fin de proveer un reacondicionamiento muscular y cardiovascular. A nivel cardiovascular, se realizan ejercicios en cicloergómetro y banda sin fin con el fin de aumentar la condición aeróbica a una intensidad elevada, a nivel muscular se realizan ejercicios de tipo isométrico e isocinético en miembros superiores e inferiores. Adicionalmente se trabaja con un sistema de poleas para entrenamiento de marcha y recuperación de fuerza durante la marcha; según la evidencia se recomienda un periodo de entrenamiento de 2 meses, con una frecuencia de 6-7 días por semana y una duración de 2 horas, adicionalmente se debe tener una dieta especial para prevenir la pérdida de masa muscular ${ }^{(2,}$ 23-26).

Tabla 1. Alteraciones fisiológicas y protocolo de entrenamiento

\begin{tabular}{|c|c|}
\hline ciones fisiológicas & Protocolo de ent \\
\hline & \multirow{5}{*}{$\begin{array}{l}\text { 1. Entrenamiento cardiovascular a } \\
\text { una intensidad alta } \\
\text { 2. Entrenamiento muscular de } \\
\text { miembros superiores e inferiores } \\
\text { 3. Entrenamiento de agilidad, } \\
\text { velocidad coordinación y balance } \\
\text { 4. Frecuencia } 6 \text { y } 7 \text { días por semana }\end{array}$} \\
\hline & \\
\hline Er & \\
\hline & \\
\hline $\begin{array}{l}\text { entllacior } \\
\text { aseoso }\end{array}$ & \\
\hline
\end{tabular}

\section{DISCUSIÓN}

La medicina aeroespacial es un campo investigado en toda su magnitud, alrededor de la evidencia se pudo identificar que existen cambios fisiológicos como la exposición a la microgravedad, las fuerzas $G$ y la presurización. Consecuentemente esta exposición trae alteraciones en los sistemas cardiovascular y muscular por lo cual se deben desarrollar programas de 
entrenamiento pre y post de misiones aeroespaciales con el fin de preparar a los astronautas para las misiones y posterior regreso a la tierra ${ }^{(27-29)}$.

El objetivo del entrenamiento físico en astronautas radica en la promoción y prevención debida a que una correcta evaluación y control de las condiciones del piloto garantiza una misión exitosa, además estos programas logran prevenir el desacondicionamiento físico de pilotos espaciales. Se debe hacer hincapié en la importancia de la estandarización de un protocolo de entrenamiento físico para pilotos espaciales, los autores destacan la importancia del trabajo en componentes cardiovasculares, musculares y sensoriales con el fin de preparar al piloto para el cambio de atmosfera ${ }^{(30-34)}$.

\section{LIMITACIONES Y CAMINOS FUTUROS}

La medicina aeroespacial es un campo que debe ser explorado por distintas áreas del saber. En fisioterapia este campo de investigación debe ser investigado con el fin de generar protocolos de entrenamiento para pilotos aeroespaciales y maximizar las capacidades físicas de los pilotos para garantizar misiones exitosas.

\section{CONCLUSIONES}

La exposición a un medio aeroespacial genera consecuencias en los sistemas cardiovascular y muscular generando atrofia muscular, alteración de la mecánica corporal y alteraciones hemodinámicas. Se debe generar un protocolo de entrenamiento para pilotos aeroespaciales con el fin de prevenir las consecuencias de los viajes aeroespaciales.

\section{REFERENCIAS}

1. Nunneley SA, Webb JT. Aerospace medicine at Brooks AFB, TX: hail and farewell. Aviat Space Environ Med. 2011;82(5):567-70.

2. Payne MW, Williams DR, Trudel G. Space flight rehabilitation. Am J Phys Med Rehabil. 2007;86(7):583-91.

3. Hawkey A. The importance of exercising in space. Interdiscip Sci Rev. 2003;28(2):130-8.

4. Hawkey $\mathrm{A}$. The physical price of a ticket into space. $\mathrm{J} \mathrm{Br}$ Interplanet Soc. 2003;56(5-6):152-9.

5. Wagner MS, Garcia K, Martin DS. Point-of-care ultrasound in aerospace medicine: known and potential applications. Aviat Space Environ Med. 2014;85(7):7309.

6. Arya R, Barnes JA, Hossain U, Patel RK, Cohen AT. Long-haul flights and deep vein thrombosis: a significant risk only when additional factors are also present. $\mathrm{Br} \mathrm{J}$ Haematol. 2002;116(3):653-4.

7. Hosoi Y, Geroulakos G, Belcaro G, Sutton S. Characteristics of deep vein thrombosis associated with prolonged travel. Eur $J$ Vasc Endovasc Surg. 2002;24(3):235-8.

8. Bagshaw M. Traveller's thrombosis: a review of deep vein thrombosis associated with travel. The Air Transport Medicine Committee, Aerospace Medical Association. Aviat Space Environ Med. 2001;72(9):848-51. 
9. Evetts SN, Caplan N, Debuse D, Lambrecht G, Damann V, Petersen N, et al. Post space mission lumbo-pelvic neuromuscular reconditioning: a European perspective. Aviat Space Environ Med. 2014;85(7):764-5.

10. Ohshima H. Musculoskeletal rehabilitation and bone. Musculoskeletal response to human space flight and physical countermeasures. Clin Calcium. 2010;20(4):537-42.

11. Convertino VA. Lower body negative pressure as a tool for research in aerospace physiology and military medicine. J Gravit Physiol. 2001;8(2):1-14.

12. Harsch V. Aerospace medicine in Germany: from the very beginnings. Aviat Space Environ Med. 2000;71(4):447-50.

13. Harsch V. Ludolph Brauer, German aeromedical pioneer. Aviat Space Environ Med. 2004;75(8):705-7.

14. Oxenkrug GF, Requintina PJ. Melatonin and jet lag syndrome: experimental model and clinical implications. CNS Spectr. 2003;8(2):139-48.

15. Thornton WE, Bonato F. Space motion sickness and motion sickness: sym ptoms and etiology. Aviat Space Environ Med. 2013;84(7):716-21.

16. Thornton WE. A rationale for space motion sickness. Aviat Space Environ Med. $2011 ; 82(4): 467-8$.

17. Harsch V. Theodor Benzinger, German pioneer in high altitude physiology research and altitude protection. Aviat Space Environ Med. 2007;78(9):906-8.

18. Gan WH, Low R, Singh J. Aviation Medicine: global historical perspectives and the development of Aviation Medicine alongside the growth of Singapore's aviation landscape. Singapore Med J. 2011;52(5):324-9.

19. Atzema C, Poirier V. Career options in aerospace and aviation medicine. Ann Emerg Med. 2004;43(5):652-6.

20. John-Baptiste A, Cook T, Straus S, Naglie G, Gray G, Tomlinson G, et al. Decision analysis in aerospace medicine: costs and benefits of a hyperbaric facility in space. Aviat Space Environ Med. 2006;77(4):434-42; discussion 42-3.

21. Wilder-Smith A, Mustafa FB, Peng CM, Earnest A, Koh D, Lin G, et al. Transient immune impairment after a simulated long-haul flight. Aviat Space Environ Med. 2012;83(4):418-23.

22. Laughlin MS, Guilliams ME, Nieschwitz BA, Hoellen D. Functional Fitness Testing Results Following Long-Duration ISS Missions. Aerosp Med Hum Perform. 2015;86(12 Suppl):A87-91.

23. Gopalakrishnan R, Genc KO, Rice AJ, Lee SM, Evans HJ, Maender CC, et al. Muscle volume, strength, endurance, and exercise loads during 6-month missions in space. Aviat Space Environ Med. 2010;81(2):91-102.

24. English KL, Lee SM, Loehr JA, Ploutz-Snyder RJ, Ploutz-Snyder LL. Isokinetic Strength Changes Following Long-Duration Spaceflight on the ISS. Aerosp Med Hum Perform. 2015;86(12 Suppl):A68-77.

25. Loehr JA, Guilliams ME, Petersen N, Hirsch N, Kawashima S, Ohshima H. Physical Training for Long-Duration Spaceflight. Aerosp Med Hum Perform. 2015;86(12 Suppl):A14-23.

26. Hackney KJ, Scott JM, Hanson AM, English KL, Downs ME, Ploutz-Snyder LL. The Astronaut-Athlete: Optimizing Human Performance in Space. J Strength Cond Res. 2015;29(12):3531-45.

27. lamenskov VV, Khafizov NN, Morozov AV. Aviation medicine: yesterday, today and tomorrow. Voen Med Zh. 2011;332(4):59-61.

28. Blaginin AA, Lizogub IN. Medical aspects of current flight safety concept of air forces of the Russian Federation. Voen Med Zh. 2012;333(12):24-6.

29. Ushakov IB, Bubeev lu A. Aviation medicine-centennial. Aviakosm Ekolog Med. 2009;43(5):3-7.

30. Jones DR, Marsh RW. Psychiatric considerations in military aerospace medicine. Aviat Space Environ Med. 2001;72(2):129-35. 
31. Williams D, Kuipers A, Mukai C, Thirsk R. Acclimation during space flight: effects on human physiology. Cmaj. 2009;180(13):1317-23.

32. Mittmeyer-Riehl M. The general practitioner of European astronauts. MMW Fortschr Med. 2016;158(8):36-7.

33. Messerschmid E, Haignere JP, Damian K, Damann V. EAC training and medical support for International Space Station astronauts. ESA Bull. 2000;104:101-8.

34. Ushakov IB, Poliakov AV, Usov VM, Boiarintsev VV. The prospects for using simulation technologies on manned spacecraft for effective training and refreshing cosmonauts' skills in providing the emergency medical aid. Aviakosm Ekolog Med. 2015;49(2):50-4.

\section{Referencias totales citadas: 34}

Referencias citadas correspondientes a la Rev Ib CC Act Fis Dep: 0 